\title{
ADAPTIVE 4D PSI-BASED CHANGE DETECTION
}

\author{
C. H. Yang *, U. Soergel \\ Institute for Photogrammetry, University of Stuttgart, Stuttgart, Germany - (yang, soergel)@ifp.uni-stuttgart.de
}

Commission III, WG III/2, III/3, III/7

KEY WORDS: Persistent Scatterer Interferometry (PSI), Synthetic Aperture Radar (SAR), Change Detection, Time Series Analysis, Urban Monitoring

\begin{abstract}
:
In a previous work, we proposed a PSI-based 4D change detection to detect disappearing and emerging PS points (3D) along with their occurrence dates (1D). Such change points are usually caused by anthropic events, e.g., building constructions in cities. This method first divides an entire SAR image stack into several subsets by a set of break dates. The PS points, which are selected based on their temporal coherences before or after a break date, are regarded as change candidates. Change points are then extracted from these candidates according to their change indices, which are modelled from their temporal coherences of divided image subsets. Finally, we check the evolution of the change indices for each change point to detect the break date that this change occurred. The experiment validated both feasibility and applicability of our method. However, two questions still remain. First, selection of temporal coherence threshold associates with a trade-off between quality and quantity of PS points. This selection is also crucial for the amount of change points in a more complex way. Second, heuristic selection of change index thresholds brings vulnerability and causes loss of change points. In this study, we adapt our approach to identify change points based on statistical characteristics of change indices rather than thresholding. The experiment validates this adaptive approach and shows increase of change points compared with the old version. In addition, we also explore and discuss optimal selection of temporal coherence threshold.
\end{abstract}

\section{INTRODUCTION}

Human activities, such as population growth, economic globalization, urban extension, and natural disasters have led to frequent urban changes. Such changes often regard building erection and demolition. Monitoring such changes is important for municipal administration. In contrast to conventional field survey, remote sensing is a fast and cost-effective alternative. Spaceborne SAR sensors provide radar images acquired rapidly over vast areas at fine spatiotemporal resolution. For instance, TerraSAR-X scans a scene size of $50 \mathrm{~km} \times 30 \mathrm{~km}$ every 11 days under a spatial resolution of $3 \mathrm{~m}$ (Stripmap mode). In addition, the active microwave sensors are capable of day-and-night vision regardless of weather conditions. These advantages make SAR suitable for monitoring tasks on this earth.

Persistent scatterer interferometry (PSI) (Crosetto et al., 2016; Ferretti et al., 2000, 2001, 2011; Hooper et al., 2004; Kampes, 2006) is designed to detect and analyse PS points based on time series analysis of multi-temporal SAR images. A temporal coherence reflects the phase stability of a pixel and can be used as an indicator for PS selection. In an urban scene, the PS points are regarded as steady structures, which persistently reflect coherent radar signals back to SAR sensors. Certain PS properties, e.g., temporal coherence, deformation velocity, and topography height, are derived for further applications.

In a previous work, we proposed a 4D change detection technique (self-citation) to recognize temporary PS points, which disappear or emerge at certain times. We distinguish and label these two point types as disappearing big change (DBC) and emerging big change (EBC) points. They are considered to be structural changes in built-up areas. This method first divides an entire SAR image stack into several subsets by a set of break dates. The PS points, which are selected based on their temporal coherences before or after a break date, are regarded as change candidates. Change points are then extracted from these candidates according to their change indices, which are modelled from their temporal coherences of divided image subsets. Finally, we check the evolution of the change indices for each change point to detect the break date that this change occurred. This technique proved to perform well for simulated and real data tests.

Two questions still remain in our previous approach. First, selection of temporal coherence threshold associates with a tradeoff between quality and quantity of PS points. How to select a suitable threshold in our method becomes even more complicated. On the one hand, raising a threshold decreases PS density as expected; on the other hand, the number of change points is reduced or increased? And how about the quality of change points being detected? Does a stricter threshold lead to more precise change detection? Second, heuristic selection of change index thresholds brings vulnerability and causes loss of change points

In this study, we propose an adaptive method to extract change points based on statistical characteristics of change indices instead of a heuristic way. In addition, we also refine the statistical model to be more sophisticated and so improve the detection accuracy. Our experiment validates our refined approach and shows increase of change points compared with the old version. In addition, we also explore and discuss optimal selection of temporal coherence threshold.

This paper is organized as follows. We describe the PSI processing and proposed method in chapters 2 and 3 . The real data test is conducted in chapter 4 to validate our method. We then experiment on and discuss several examples of different

\footnotetext{
* Corresponding author
} 
temporal coherence thresholds in chapter 5 . Finally, chapter 6 summarizes the conclusions and future works.

\section{PERSISTENT SCATTERER INTERFEROMETRY}

The input data are a time series of $N$ spatially overlapped SAR images acquired from the same orbit. $N$-1 slave images are precisely co-registered to a master image, which is selected under small baseline constraint (Berardino et al., 2002; Lanari et al., 2004). Then, $N-1$ interferograms between the master and all of the slave images are computed. The interferometric phases of each pixel are used to estimate its temporal coherence (Ferretti et al., 2001), line-of-sight velocity, and relative topography height. The temporal coherence serves as a measure of phase stability throughout the SAR images. Finally, those pixels with high temporal coherences are selected as PS points. However, DBC and EBC points, if any, are just discarded here as they suffer coherence loss during the entire image sequence. To retrieve such big change information, we resort to our approach described in the next section.

\section{METHODOLOGY}

\subsection{Single-break-date Scheme}

We first illustrate the change detection scheme (Figure 1) subject to a single break date that big changes occur before or after. The complete set consists of an entire image set. The front and back sets comprise the images taken before and after the break date, respectively. Our aim is to find change points, which exist as PS points in the front set but disappear in the back set and vice versa (emergence). The three image sets are processed by a standard PSI procedure to generate three temporal coherence images. Two change index images are calculated by subtracting the temporal coherence image of the complete set from the ones of the front and back sets. The change indices are used to extract the change candidates. These candidates are jointly analysed with the PS points, which are selected from the complete set, to determine the final point labels. Finally, the PS and change points are combined into a change detection result.

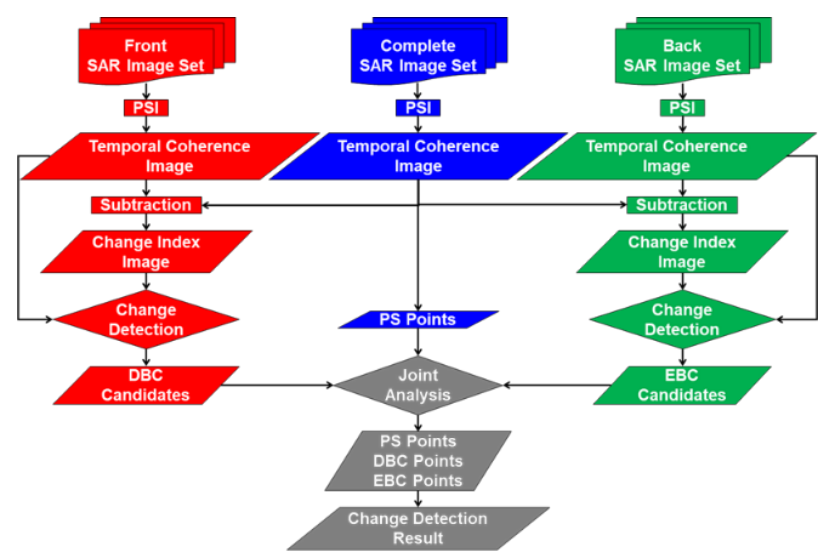

Figure 1. Flowchart of single-break-date change detection. Persistence (blue), disappearance (red), and emergence (green) scenarios are dedicated to extracting PS, DBC, and EBC points, respectively.

\subsection{Change Index}

We introduce a change index derived by temporal coherence to quantify each pixel's probability of being a change point at a break date. Our approach assumes that the temporal coherence estimates of a PS point in complete, front, and back sets are approximately the same. In contrast, the temporal coherence of a change point in a front or back set is higher than that in a complete set (coherence loss due to the change). Based on these assumptions, the change indices of a pixel in disappearance $\left(\mathrm{CI}^{\mathrm{D}}\right)$ and emergence $\left(C I^{\mathrm{E}}\right)$ scenarios are calculated by

$$
\begin{gathered}
\underset{[-1,+1] \in \mathrm{R}}{C I^{\mathrm{D}}}=\gamma^{\mathrm{F}}-\gamma^{\mathrm{C}} \\
\underset{[-1,+1] \in \mathrm{R}}{C I^{\mathrm{E}}}=\gamma^{\mathrm{B}}-\gamma^{\mathrm{C}}
\end{gathered}
$$

where $\gamma^{\mathrm{C}}, \gamma^{\mathrm{F}}$, and $\gamma^{\mathrm{B}}$ denote temporal coherences in complete, front, and back sets. A pixel is more likely to be a DBC or EBC point when $C I^{\mathrm{D}}$ or $C I^{\mathrm{E}}$ more tends towards 1 , respectively. Otherwise, change indices of PS points concentrate towards 0 .

\subsection{Change Detection}

Based on change indices, we design a statistical-based method to extract change points. Given that no big change occurs, a change index distribution over PS points is assumed to follow a Gaussian distribution

$$
\mathrm{N}\left(C I_{\mathrm{PS}} \mid \mu, \sigma\right)=\frac{1}{\sigma \sqrt{2 \pi}} \exp \left(\frac{-C I_{\mathrm{PS}}^{2}}{2 \sigma^{2}}\right) .
$$

The mean $\mu$ of change indices is anticipated to be 0 . However, a systematic positive bias is likely to occur due to overestimation of temporal coherence from the shorter front or back set in contrast to the complete set. In contrast to PS points, a change index distribution over change points does not conform to the Gaussian model because the big changes substantially and arbitrarily alter their temporal coherences between complete, front, and back sets. The significant difference between the change index distributions over PS and change points plays a major role to separate these two point types.

We first select PS points from a front or back set and sketch their change index distribution (red curve, Figure 2). Parts of these PS points disappear or emerge in the complete set and therefore turn into change points; the others remain the same as PS points. This change index distribution is modelled as the sum of a Gaussian curve plus an asymmetric probability distribution function of a large right tail. The Gaussian curve originates from the change indices of the PS points that remain in the complete set; the right tail is caused mainly by the high change indices of the change points. In our previous method (self-citation) we fitted the Gaussian curve (blue curve) by a model-driven way and employed its $3 \sigma$ value as a threshold to extract the change points. However, we unavoidably missed some of the change points. To solve this problem, we resort to a new way in the following.

We observe a systematic positive bias of the change index distribution (Figure 2). For this reason, we can no longer regard change indices near 0 as an indication of PS points. To compensate for this bias, we shift this indication to the value 0.045 subject to the peak and consider it as a threshold for change candidates (change indices on the right side). We then check whether the change candidates are PS points in the complete set. If so, they are classified as PS points; otherwise, we label them as change points.

The single-break-date scheme has three limitations. First, detection of big changes is dependent on a break date, which can be manually set for specific interests. This requirement restricts the applicability particularly when a priori knowledge of scene changes is unavailable. Second, accurate occurrence times of big 
changes are lacking as they are only known to disappear or emerge after a break date. Last but not least, false labels might happen because of uncertainty and imperfection during PSI processing. We then combine several single-break-date results to overcome these limitations.

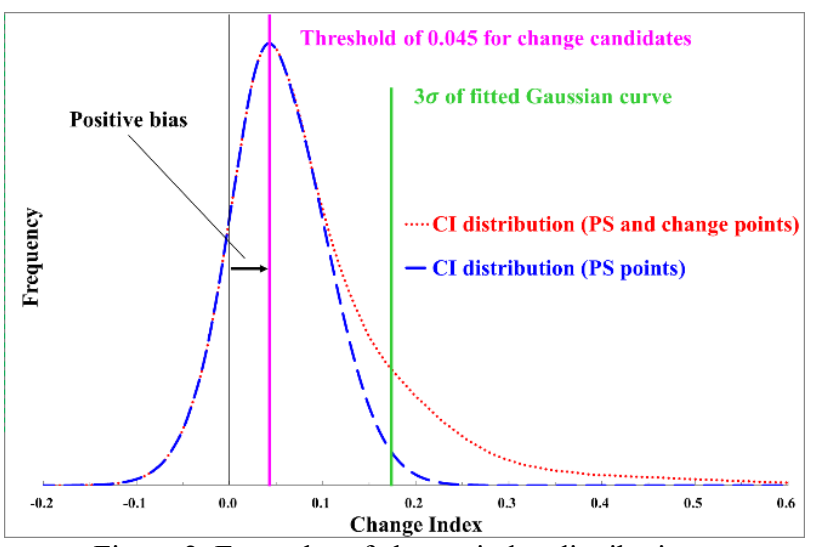

Figure 2. Examples of change index distributions.

\subsection{Multi-break-date Change Detection}

The multi-break-date scheme (Figure 3) demands a set of singlebreak-date results as input, which are subject to time-series break dates. Each pixel contains a sequence of change indices and the corresponding initial point labels (PS, DBC, or EBC). A joint analysis is applied to each pixel's initial point labels to determine its final label. To begin with, a pixel is labelled as PS if all of its initial point labels are PS. In contrast, a pixel initially labelled as $\mathrm{DBC}$ or EBC is labelled as such. If both initial labels coincide, we utilize voting to determine the final label. Afterwards, the occurrence date of each change point is detected from the timeseries break dates based on the temporal variation in its change index sequence. In the end, the PS and change points along with the occurrence dates are combined to illustrate the spatiotemporal results.

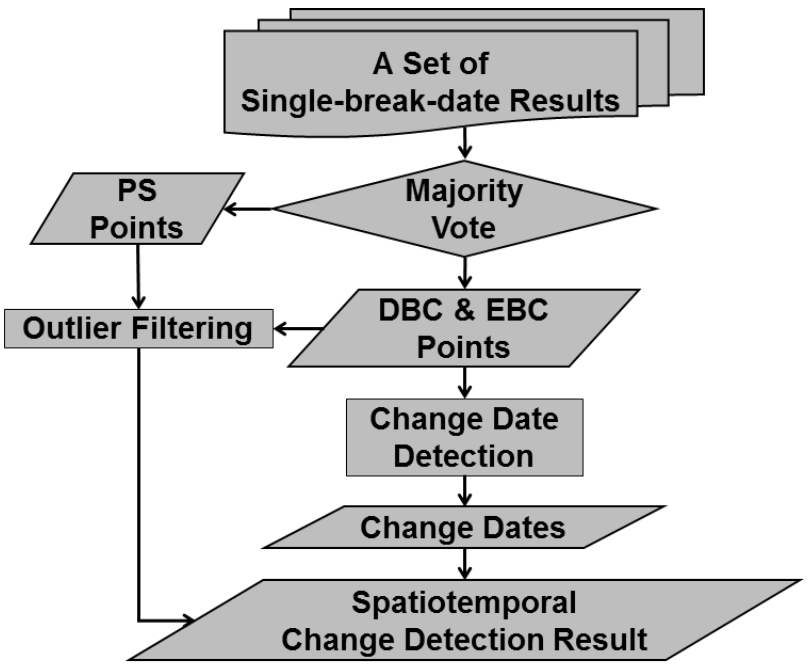

Figure 3. Flowchart of multi-break-date change detection.

\subsection{Detection of Events' Dates}

Consider a DBC point first, we simulate a typical evolution of change indices calculated by (1) for such case (Figure 4). The disappearance date is right on the turning point. The reason is that the temporal coherences $\gamma^{\mathrm{F}}$ in the front image set decreases gradually after the disappearance. We adopt a simple geometric algorithm to detect this turning point. First, a horizontal line 1 extends from the sequence beginning to the left. Starting from the terminal of line 1 , a straight line 2 is drawn to the end of the sequence. The turning point, which features the longest distance (line 3) to line 2, can then be detected. Finally, the corresponding break date is regarded as the disappearance date. We adopt a similar process to detect emergence dates of EBC points as well.

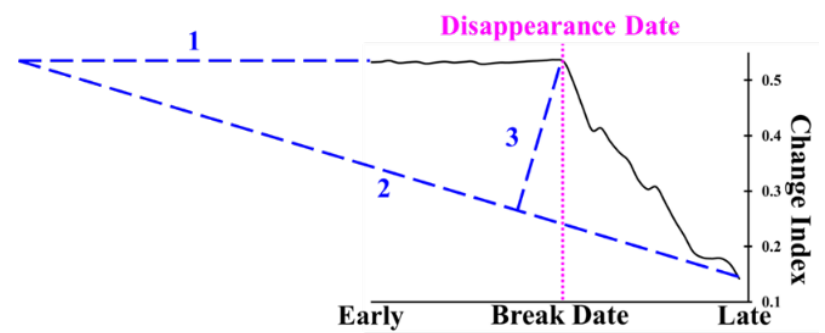

Figure 4. Change index sequence pertaining to disappearance.

\section{REAL DATA TEST}

We focus our discussion on the area of interest at the north of Berlin central station (Figure 5). The mean TerraSAR-X image (Figure 5(a)) shows many bright clusters of strong signals, which appear to be potential PS and change points. The yellow squares in the aerial image (Figure 5(b)) indicates the construction events taking place in 2013. We adopted forty TerraSAR-X images (Table 1) for our test, which were acquired in High Resolution Spotlight mode from October 27, 2010 to September 4, 2014. All of the images were precisely co-registered and resampled into $5000 \times 5000$ grid (ground resolution: $1 \mathrm{~m})$. The thirteen break dates ( $b d: 16$ to 28 ) were chosen to detect the ground changes within 2013.

Our result successfully detects the construction events along with their occurrence dates (Figure 6). We have proven that the detected events and times all agree with the ground truth in the previous study (self-citation). The improvement here is that the DBC and EBC points are increased by $12 \%$ and $29 \%$, respectively. These additional change points are those missed due to the heuristic thresholding of the previous method.

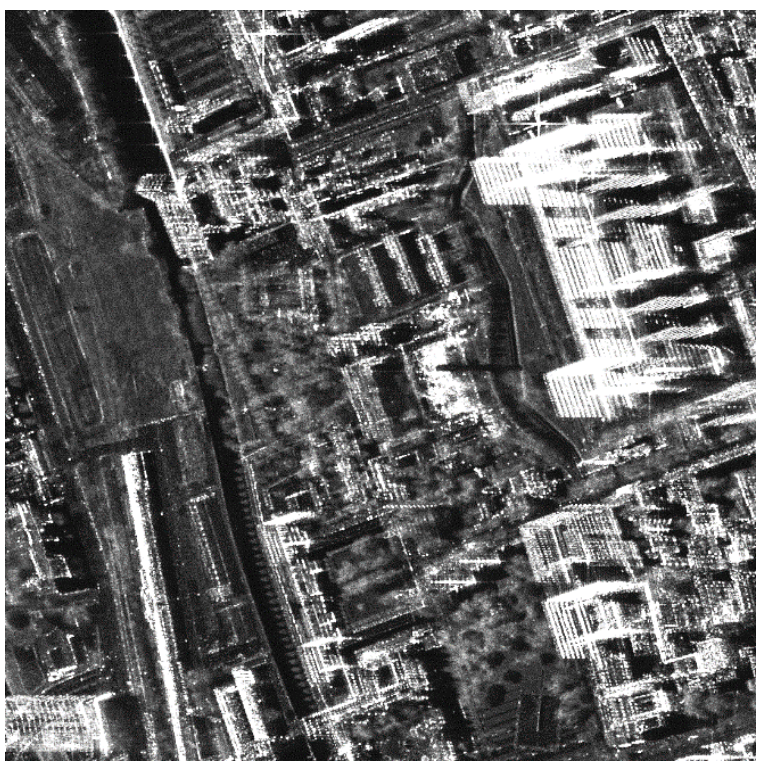

(a) 


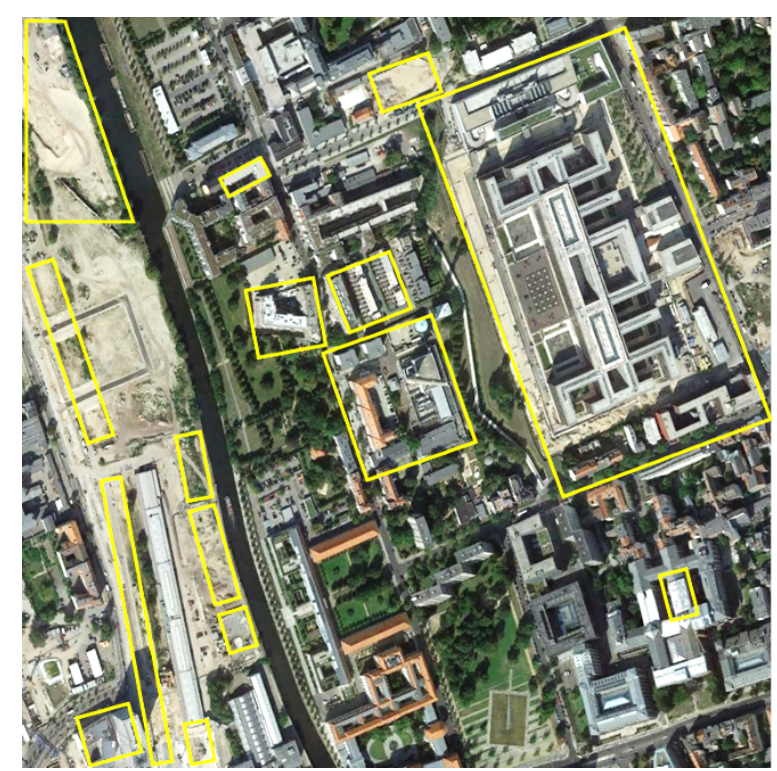

(b)

Figure 5. Study area at the north of Berlin central station. (a) Mean TerraSAR-X image. (b) Aerial image (Google Earth) acquired on September 5, 2014. Yellow square, building construction.

In this test, we adopted the coherence threshold of 0.8 to select the PS and change candidates. Such strict threshold is supposed to bring reliable PS and change points but also retain sufficient quantity of intact information for built-up areas. However, we did not verify this supposition before. We will compare and analyse the results of different thresholds via both quantitative and qualitative ways.

\begin{tabular}{|c|c|c|c|}
\hline \multicolumn{4}{|c|}{ Acquisition Dates of TSX Images } \\
\hline 2010/10/27 & 2011/08/31 & $2013 / 08 / 26$ & 2014/02/07 \\
\hline $2010 / 11 / 18$ & $2011 / 10 / 03$ & $\begin{array}{c}b d=21 \\
2013 / 09 / 17\end{array}$ & $2014 / 03 / 01$ \\
\hline & & $b d=22$ & \\
\hline 2011/01/23 & 2011/12/30 & $2013 / 09 / 28$ & $2014 / 03 / 23$ \\
\hline & & $b d=23$ & \\
\hline 2011/02/14 & 2012/01/10 & $2013 / 10 / 20$ & 2014/05/06 \\
\hline & & $b d=24$ & \\
\hline 2011/03/08 & 2012/02/01 & 2013/10/31 & $2014 / 05 / 28$ \\
\hline 2011/03/30 & 2012/02/12 & $2013 / 11 / 22$ & 2014/06/19 \\
\hline & $b d=16$ & $b d=26$ & \\
\hline 2011/06/04 & $2013 / 06 / 21$ & $2013 / 12 / 03$ & 2014/07/11 \\
\hline & $b d=17$ & $b d=27$ & \\
\hline 2011/06/15 & 2013/07/13 & $2013 / 12 / 25$ & 2014/08/02 \\
\hline & $b d=18$ & $b d=28$ & \\
\hline 2011/07/18 & 2013/07/24 & 2014/01/05 & 2014/08/24 \\
\hline 2011/08/20 & $\begin{aligned} & b d=19 \\
& 2013 / 08 / 15\end{aligned}$ & 2014/01/16 & 2014/09/04 \\
\hline
\end{tabular}

Table 1. TerraSAR-X images and break date $(b d)$ setting.

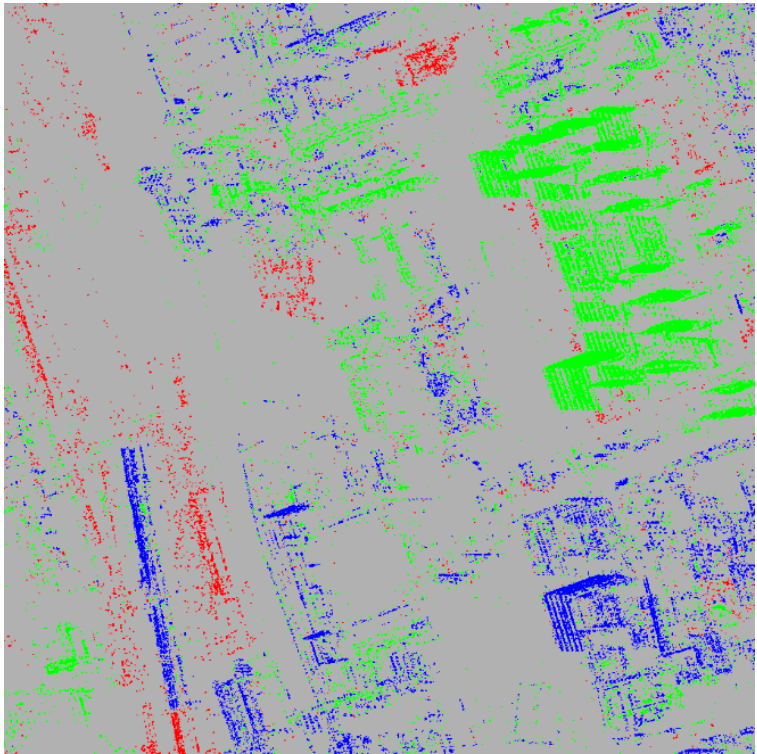

(a)

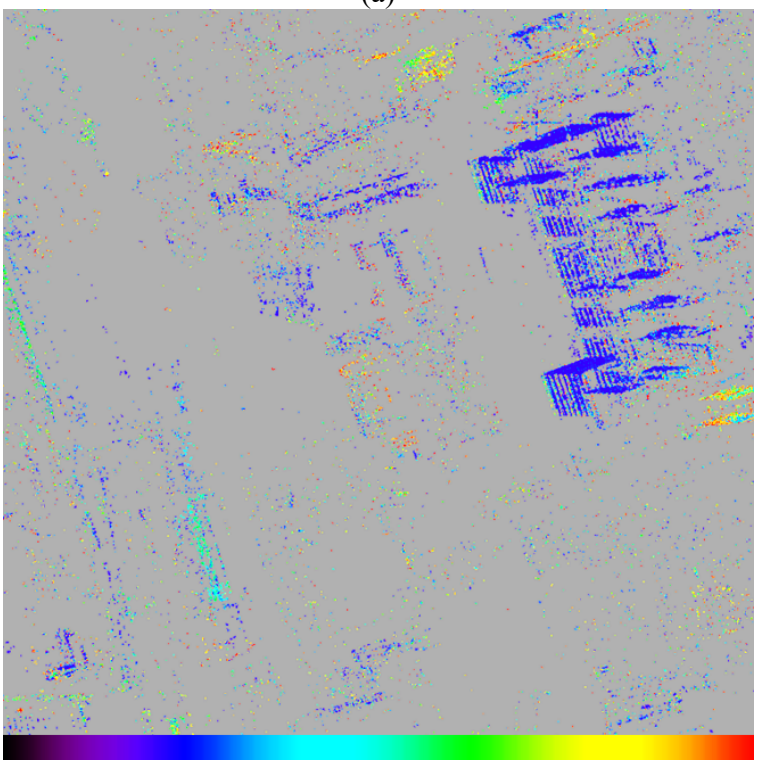

(b)

Figure 6. Spatiotemporal change detection result. (a) Steady, disappearing, and emerging structures represented by PS (blue), DBC (red), and EBC (green) points. (b) Occurrence dates: black to red, earliest to latest in 2013.

\section{OPTIMAL SELECTION OF TEMPORAL COHERENCE THRESHOLD}

We first look into the correlation between the temporal coherence thresholds (from 0.4 to 0.9 ) and the quantities of the PS and change points (Figure 7). The PS density slides down as a smooth quadratic curve when the threshold is raised. The DBC density reaches the maximum at the threshold of 0.6 , after which it becomes sparser gradually. We also observe a similar but more drastic course for the EBC density. Now we turn to the change detection results (Figure 8) to discuss the accuracy. In the beginning (threshold of 0.4 ), the scene is overwhelmed by those PS points of low quality, which contradicts our ground truth (Figure 5(b)). As the threshold is increased, the ratio of PS to change points turns reversely. The patterns of the clustered change points become more and more significant while the false PS points are filtered out. This phenomenon illustrates that raising a threshold can identify more authentic PS and change 
points. Nevertheless, the cost is to bring more false change points. We see that the extreme example (Figure 8(e), threshold of 0.6) contains the intensive change points everywhere. When the threshold $(>0.6)$ is set larger and larger, the number of the false change points (outside the change areas) decreases in a larger scale; in contrast, more and more true ones (inside the change areas) are detected. Although a high threshold seems promising for accuracy, we also notice that the quantity of both the PS and change points is overly underestimated in particular when the threshold is larger than 0.8. By comparison, the loss of the change points is more restrained. The reason is that our methodology offers more opportunities of being change points, which depends on how many break dates are employed. We conclude that the optimal result in our case comes out with the threshold of 0.8 . We suggest this threshold of 0.8 for urban scenes and high-resolution SAR images.

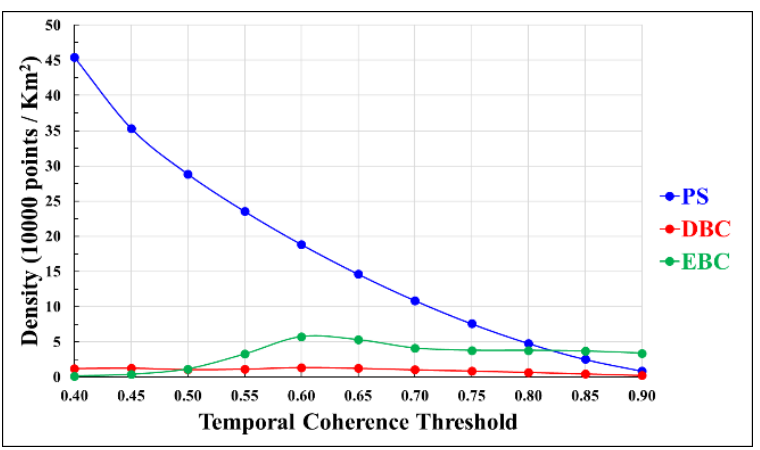

(a)

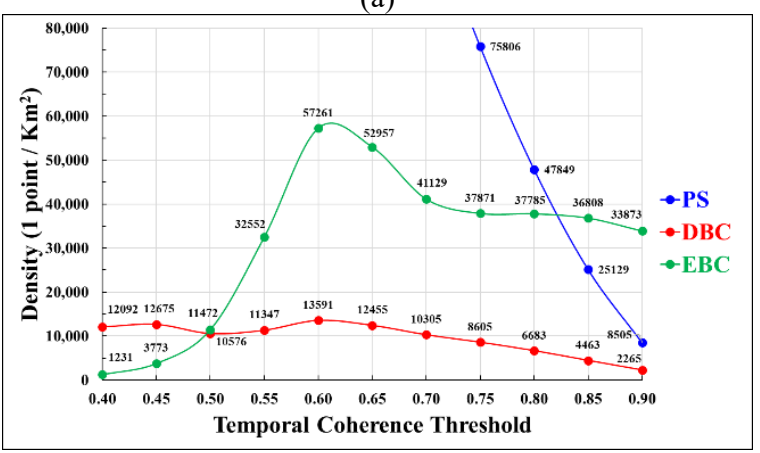

(b)

Figure 7. Point density versus temporal coherence threshold. (a) and (b), small and large scales.

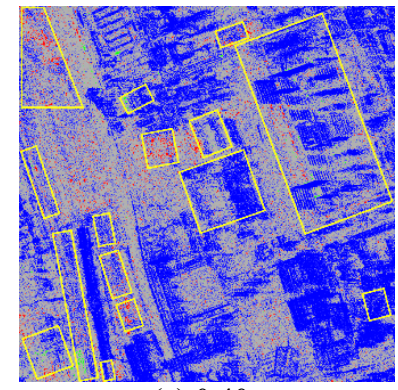

(a) 0.40

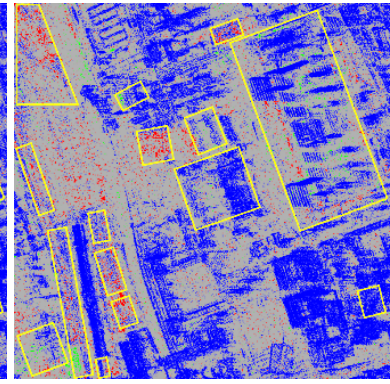

(b) 0.45

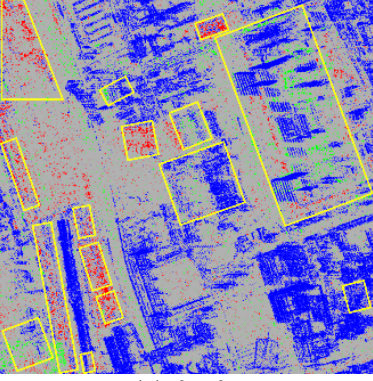

(c) 0.50

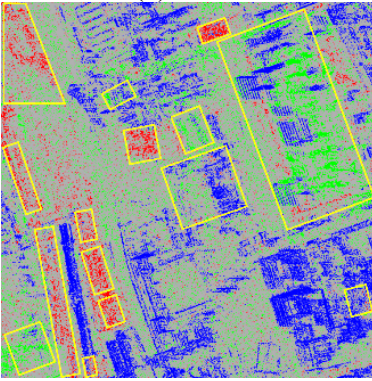

(e) 0.60

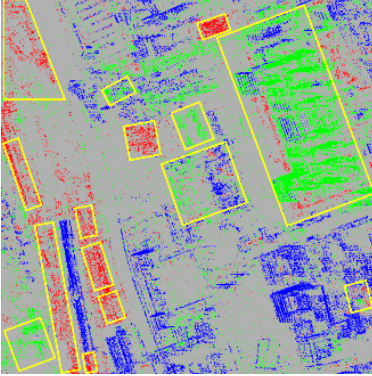

(g) 0.70

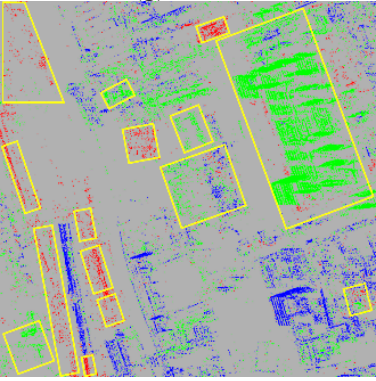

(i) 0.80

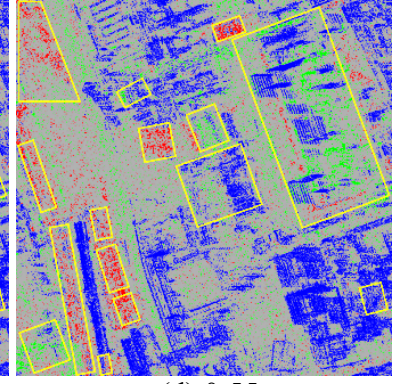

(d) 0.55

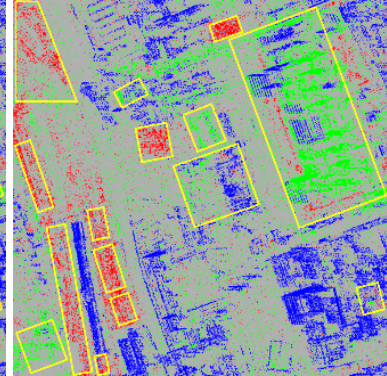

(f) 0.65

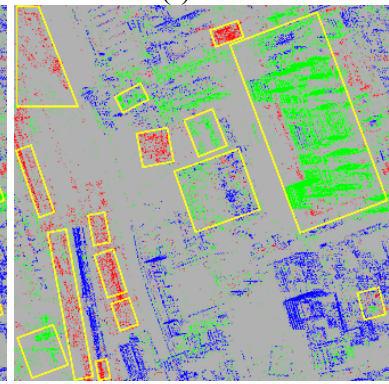

(h) 0.75

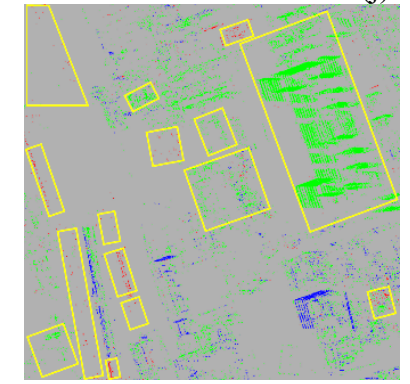

(k) 0.90

Figure 8. Change detection results subject to temporal coherence thresholds (from 0.4 to 0.9 ). Steady, disappearing, and emerging structures represented by PS (blue), DBC (red), and $\mathrm{EBC}$ (green) points. Yellow square, building construction.

\section{CONCLUSIONS}

Earlier, we proposed an approach to detect disappearing and emerging PS points. Such change points are regarded as structural changes in urban areas. The core of this method was to 
introduce a change index based on temporal coherence to quantify each pixel's probability of being a change point. Pixels are labelled as change candidates if their temporal coherences over a specific time span fulfil a specified threshold. We then used a heuristic change index threshold to extract change points from the candidates. However, some change points were missed unavoidably.

In this paper, we explore the statistical characteristics of change indices from PS and change points. The statistical difference between these two point types is then used to identify and separate them. Without heuristic thresholding, the change points can be fully detected. Our real data test proves that the numbers of the disappearing and emerging points are increased by $12 \%$ and $29 \%$, respectively. In addition, we also explore the influence of temporal coherence threshold on change detection results. Generally speaking, loose thresholds under a certain level cause a considerable amount of false PS and missing change points. When the threshold is increasing, we get rid of more false PS points and meanwhile retrieve more change points. However, use of a too strict threshold costs too much information loss of both point types (especially for PS points). We recommend a threshold of 0.8 when urban scenes and high-resolution SAR images are considered.

In the future, we will upgrade our technique to detect a new change point label, which undergoes double big changes during a time sequence. For instance, a new building is erected soon, following a demolition event. To do so, we must further look into the change index sequence of one single pixel if its initial pint labels contain different change labels. Regarding applications, we will focus our study area on Stuttgart, Germany. Since 2010, many constructions, e.g., the main station, buildings, and traffic lines, proceeded and require cost-effective monitoring approaches for municipal administration. For this purpose, we will test and assess our method with cost-free Sentinel-1 images.

\section{REFERENCES}

Berardino, P., Fornaro, G., Lanari, R. and Sansosti, E., 2002. A new algorithm for surface deformation monitoring based on small baseline differential SAR interferogram. IEEE Transactions on Geoscience and Remote Sensing, 40(11), pp. 2375-2382.

Crosetto, M., Monserrat, O., Cuevas-González, M., Devanthéry, N. and Crippa, B., 2016. Persistent scatterer interferometry: a review. ISPRS Journal of Photogrammetry and Remote Sensing, 115 , pp. $78-89$.

Ferretti, A., Prati, C. and Rocca, F., 2000. Nonlinear subsidence rate estimation using permanent scatterers in differential SAR interferometry. IEEE Transactions on Geoscience and Remote Sensing, 38(5), pp. 2202-2212.

Ferretti, A., Prati, C. and Rocca, F., 2001. Permanent scatterers in SAR interferometry. IEEE Transactions on Geoscience and Remote Sensing, 39(1), pp. 8-20.

Ferretti, A., Fumagalli, A., Novali, A., Prati, C., Rocca, F. and Rucci, A., 2011. A new algorithm for processing interferometric data-stacks: SqueeSAR. IEEE Transactions on Geoscience and Remote Sensing, 49(9), pp. 3460-3470.

Hooper, A., Zebker, H., Segall, P. and Kampes, B., 2004. A new method for measuring deformation on volcanoes and other natural terrains using InSAR persistent scatterers. Geophysical Research Letters, 31(23), pp. 1-5.
Kampes, B. M., 2006. Radar Interferometry: Persistent Scatterer Technique. Springer.

Lanari, R., Mora, O., Manunta, M., Mallorquí, J. J., Berardino, P. and Sansosti, E., 2004. A small baseline approach for investigating deformations on full resolution differential SAR interferograms. IEEE Transactions on Geoscience and Remote Sensing, 42(7), pp. 1377-1386. 